Pacific Journal of Mathematics

ISOLATION AMONGST THE COMPOSITION OPERATORS Joel Harold Shapiro and CarL Sundberg 


\title{
ISOLATION AMONGST THE COMPOSITION OPERATORS
}

\author{
Joel H. Shapiro AND CARl SUNDberg
}

\begin{abstract}
Earl Berkson has shown that certain highly non-compact composition operators on the Hardy space $H^{2}$ are, in the operator norm topology, isolated from all the other composition operators. On the other hand, it is easy to see that no compact composition operator is so isolated. Here we explore the intermediate territory, with the following results: (i) Only the extreme points of the $H^{\infty}$ unit ball can induce isolated composition operators. In particular, those holomorphic self-maps of the unit disc whose images make at most finite order of contact with the unit circle induce composition operators that are not isolated. However, (ii) extreme points do not tell the whole story about isolation: some of them induce compact, hence non-isolated, composition operators. Nevertheless, (iii) all sufficiently regular univalent extreme points induce isolated composition operators.
\end{abstract}

Introduction. It is a familiar fact of elementary function theory that the composition of holomorphic functions is again holomorphic. More precisely, if $\varphi$ is a holomorphic function taking a plane domain into itself, and if $f$ is holomorphic on that domain, then so is the composition $f \circ \varphi$. Less familiar is the fact that if the domain is the unit disc $U$, and $f$ belongs to the Hardy space $H^{2}$ of $U$, then so does $f \circ \varphi$. This is Littlewood's Subordination Principle ([13], [17], [25]), which in modern language states that the composition operator $C_{\varphi}$ defined on functions holomorphic in $U$ by:

$$
C_{\varphi} f=f \circ \varphi \quad(f \text { holomorphic in } U)
$$

restricts to a bounded linear operator on $H^{2}$. The remarkable aspect of Littlewood's Principle is that nothing extra is required of the holomorphic map $\varphi$ : it need not be univalent, or even boundedly valent, nor is it required to have any regularity at the boundary.

Littlewood's Principle raises the possibility of explaining the behavior of the operator $C_{\varphi}$ in terms of the function theoretic properties of the inducing map $\varphi$, and so provides a new point of contact between function theory and functional analysis. Ground in this area was broken about twenty years ago by Eric Nordgren [20], who determined the spectra of composition operators induced by disc automorphisms; 
by J. V. Ryff [23], who studied norm inequalities; and by Howard J. Schwartz [24], who (among other things) studied the compactness of composition operators. The work of these authors gave rise to subsequent studies of spectra ([6], [7], [8], [16], [20]), compactness ([18], [25], [26]), cyclicity ([4], [29]), subnormality ([8], [9], [11]), and semigroups ([1], [3], [27]), to name several of the many topics of current interest.

The work to be described here originates from two sources: the compactness studies cited above, and the following result of Earl Berkson.

Berkson's Isolation TheOREM ([2]). If $\varphi$ has radial limits of modulus 1 on a set $E \subset \partial U$ of positive measure, then for every other holomorphic self-map $\psi$ of $U$ :

$$
\left\|C_{\varphi}-C_{\psi}\right\| \geq \sqrt{\frac{\text { meas } E}{2}}
$$

The norm here is the usual one for bounded linear operators on $H^{2}$, and the measure is normalized Lebesgue measure on the unit circle. Berkson's theorem makes a topological statement about the space $\operatorname{Comp}\left(\mathrm{H}^{2}\right)$ of composition operators on $\mathrm{H}^{2}$, endowed with the operator norm metric. It says that the composition operator $C_{\varphi}$ is isolated in $\operatorname{Comp}\left(H^{2}\right)$ whenever $|\varphi|=1$ on a subset of the unit circle having positive measure. For example this result locates the identity operator, as well as any composition operator induced by an inner function, at least $1 / \sqrt{2}$ units distant from every other composition operator.

At the other extreme, an elementary argument (Proposition 2.2) shows that the compact composition operators are dramatically nonisolated: they all lie in the same path component of $\operatorname{Comp}\left(H^{2}\right)$.

The problem of characterizing the compact composition operators is a subtle one that has only recently been answered [25]. Early on, Schwartz observed that holomorphic self-maps $\varphi$ of $U$ which have radial limits of modulus one on a set of positive measure induce noncompact composition operators on $H^{2}$ ([24], see also [26]). He also observed that there are other non-compact composition operators, for example the one induced by the linear fractional map $(1+z) / 2$. In the other direction Schwartz showed that $C_{\varphi}$ is compact whenever $\varphi(U)$ has no limit points on the unit circle. The first author and P. D. Taylor extended this result by showing that $C_{\varphi}$ is compact whenever $\varphi(U)$ lies in a polygon inscribed in the unit circle ([26] Corollary 3.2; [25] sec. 2.4; and sec. 3.4 below), a result which persists if the corners 
of the polygon are rounded just a little bit ([26], Cor. 4.4). These examples suggest the following principle:

" $C_{\varphi}$ is compact $\Leftrightarrow \varphi(z)$ is not too close to $\partial U$ too often".

The precise formulation of this principle involves the value distribution theory of the map $\varphi$ ([25], Theorem 2.3). It will not figure strongly in our work here.

The purpose of this paper is to explore the ground that lies between compactness and the extremely non-compact situation of Berkson's theorem. Such a study was first proposed by Aristomenis Siskakis [28], who asked if every non-compact composition operator had to be isolated in $\operatorname{Comp}\left(H^{2}\right)$. We show here that this is not the case (Theorem 3.1): if $\varphi$ is a holomorphic self map of $U$ for which

$$
\int_{0}^{2 \pi} \log \left(1-\left|\varphi\left(e^{i \theta}\right)\right|\right) d \theta>-\infty,
$$

then $C_{\varphi}$ is not isolated in $\operatorname{Comp}\left(H^{2}\right)$. We observe that condition (*) is satisfied whenever $\varphi(U)$ makes at most finite order of contact with the unit circle, and it even allows a certain degree of "exponential contact." The details occupy $\S 3$.

Condition $(*)$ has appeared before in the theory of Hardy spaces: it characterizes those members of the unit ball of $H^{\infty}$ that are not extreme points ([13], Theorem 7.9, page 125). Here $H^{\infty}$ denotes the Banach space of bounded holomorphic functions on $U$, taken in the supremum norm. In this paper the notion of "extreme point" will serve only to signal the divergence of the integral in $(*)$. The geometric interpretation of extreme point will play no role in our work.

We will show that condition $(*)$, though sufficient for non-isolation, is not necessary: there exist extreme points (in fact univalent ones) which induce composition operators that are compact, and therefore not isolated (Theorem 3.5).

In the other direction, we show in $\S 4$ that whenever $\varphi$ is a univalent extreme point mapping $U$ onto a sufficiently regular sub-region then $C_{\varphi}$ is isolated. A major aspect of this extra regularity is a smoothness requirement on the boundary of the image.

In the interests of completeness, we record in the first section of the paper, mostly without proof, the prerequisites required from function theory and the theory of composition operators. In the second section, which serves as an introduction to the phenomenon of isolation, we relate isolation with subordination, show that the compact composition operators are not isolated, and present a simpler proof of 
Berkson's theorem, which, incidentally, provides an improved lower bound. As mentioned above, the third and fourth sections contain the main results of the paper, concerning respectively nonisolation and isolation.

Our work raises the problems of essential isolation, compact differences, and components. It turns out that the composition operators which are shown in this paper to be isolated, are actually isolated in the weaker "essentially norm topology," while each of those shown to be not isolated lies on an arc in $\operatorname{Comp}\left(H^{2}\right)$, and has compact difference with every other member of that arc. Our results suggest that two composition operators may belong to the same component of $\operatorname{Comp}\left(\mathrm{H}^{2}\right)$ if and only if they differ by a compact. We discuss these matters, along with some other open problems, in the final section.

Acknowledgments. We wish to thank Aristomenis Siskakis for suggesting the isolation problem; and Paul Bourdon, Lech Drewnowski, and Peter Lappan for helpful discussions about the material of this paper.

1. Preliminaries. In this reference section we record our notation, and sketch the prerequisites required for the rest of the paper.

1.1. Notation. As already mentioned, $U$ denotes the open unit disc. The unit circle is denoted by $\partial U$, and the letters $\varphi$ and $\psi$, with or without subscripts, always represent holomorphic functions taking $U$ into itself, the symbol $\Pi^{+}$signifies the upper half-plane, and its intersection with a subset $E$ of the complex plane is denoted by $E^{+}$. The closure (in $\mathbb{C}$ ) of the set $E$ will be denoted by $\bar{E}$.

The abbreviation "a.e." always refers to Lebesgue measure, on either the circle or the real line. We denote by $\sigma$ normalized Lebesgue arclength measure on $\partial U$.

The norm of a bounded linear operator $T$ on $H^{2}$ will be denoted by $\|T\|$ when there is no danger of confusion with the $H^{2}$ norm.

1.2. The Hardy space $H^{2}$. Details about this subject can be found in any standard reference, e.g. [13] or [22]. The Hardy space $H^{2}$ is the collection of functions $f$ holomorphic on $U$ for which

$$
\|f\|^{2} \stackrel{\text { def }}{=} \sup _{0 \leq r \leq 1} \int|f(r \zeta)|^{2} d \sigma(\zeta)<\infty
$$

According to Fatou's Theorem, each $f \in H^{2}$ has, for a.e. $\zeta \in \partial U$, a radial limit

$$
f(\zeta) \stackrel{\text { def }}{=} \lim _{r \rightarrow 1-} f(r \zeta)
$$


and it is well known that

$$
\|f\|^{2}=\int|f(\zeta)|^{2} d \sigma(\zeta)
$$

Thus the map

$$
f \rightarrow \text { radial limit function of } f
$$

is an isometry taking $H^{2}$ onto a closed subspace of $L^{2}(\partial U)$ ([22], Theorem 17.10, page 366), and this exhibits $H^{2}$ as a Hilbert space with inner product

$$
\langle f, g\rangle=\int_{\partial U} f \bar{g} d \sigma \quad\left(f, g \in H^{2}\right) .
$$

1.3. Reproducing kernels. For each point $\alpha \in U$ the reproducing kernel

$$
k_{\alpha}(z)=\frac{1}{1-\bar{\alpha} z} \quad(z \in U)
$$

belongs to $H^{2}$, and represents the linear functional of evaluation at $\alpha$ :

$$
f(\alpha)=\left\langle f, k_{\alpha}\right\rangle \quad\left(f \in H^{2}\right) .
$$

In particular,

$$
\left\|k_{\alpha}\right\|^{2}=\left\langle k_{\alpha}, k_{\alpha}\right\rangle=k_{\alpha}(\alpha)=\frac{1}{1-|\alpha|^{2}} .
$$

The last two equations give an important pointwise estimate on $H^{2}$ functions:

$$
|f(\alpha)| \leq\|f\|\left\|k_{\alpha}\right\|=\frac{\|f\|}{\sqrt{1-|\alpha|^{2}}} \quad(\alpha \in U) .
$$

This estimate shows that convergence in $H^{2}$ implies uniform convergence on compact subsets of $U$, and that bounded subsets of $H^{2}$ are relatively compact in the topology of uniform convergence on compact sets. In $\S 3$ the corresponding inequality on the derivative of $f$ will play an important role. It is most easily derived from an application of the Cauchy-Schwarz inequality to the power series representation of $f^{\prime}$.

$$
\left|f^{\prime}(\alpha)\right| \leq \frac{\|f\|}{(1-|\alpha|)^{3 / 2}} \quad(\alpha \in U) .
$$

1.4. Log-integrability and outer functions. If $f \in H^{2}$ is not identically zero, then its radial limit function cannot be too small too often. More precisely, $\log |f|$ must be integrable over the unit circle 
([13], Theorem 2.2, page 17). In fact, log-integrability characterizes the boundary moduli of $H^{2}$-functions among the square integrable functions. Suppose $g$ is a non-negative function on $\partial U$, squareintegrable with respect to $\sigma$, and suppose further that $u=\log g$ is integrable with respect to $\sigma$. Then we can extend $u$ harmonically to $U$ by the Poisson integral formula. Let $\tilde{u}$ denote the harmonic conjugate of $u$ in the unit disc. Then the function $F=\exp (u+i \tilde{u})$ belongs to $H^{2}$, and on $\partial U$ its modulus coincides a.e. with $g$ ([13], page 24).

1.5. A Phragmen-LindelöF PRINCIPLE ([13], Theorem 2.11, page 28). If the radial limit function of $f \in H^{2}$ is essentially bounded on $\partial U$, then $f$ is bounded on $U$, and

$$
\sup \{|f(z)|: z \in U\}=\operatorname{ess} \sup \{|f(\zeta)|: \zeta \in \partial U\} \quad(z \in U) .
$$

1.6. Contact with the boundary. To measure how closely subregions of the unit disc contact the boundary, we employ the class of contact functions. These are continuous, $2 \pi$-periodic functions $\kappa: \mathbb{R} \rightarrow[0,1)$ which, on the interval $[-\pi, \pi]$, decrease monotonically on the subinterval to the left of the origin, increase monotonically on the subinterval to the right, and vanish precisely at the origin. Each contact function $\kappa$ defines an approach region:

$$
\Omega(\kappa)=\left\{r e^{i \theta}: 0 \leq r<1, \theta \text { real, and } 1-r>\kappa(\theta)\right\},
$$

whose boundary, the curve described in polar coordinates by the equation

$$
r=1-\kappa(\theta) \quad(-\pi \leq \theta \leq \pi),
$$

is a Jordan curve in the closed unit disc that meets the unit circle only at the point 1 (the "vertex" of the approach region). For example, if $\kappa(\theta)=|\theta|$ on $[-\pi, \pi]$, then $\Omega(\kappa)$ is a standard nontangential approach region. More generally, the approach region $\Omega(\kappa)$ contains the interior of a triangle with vertex at 1 if and only if $\kappa(\theta) \geq \operatorname{constant}|\theta|$ on $[-\pi, \pi]$. In this case it is common practice to say that $\Omega(\kappa)$ obeys a cone condition.

Note that approach regions need not be symmetric with respect to the real axis, since the contact functions which define them are not required to be even.

It is easy to check that the boundary of an approach region inherits the smoothness of its defining contact function. For example, if $\kappa$ is of class $C^{n}$, then so is $\partial \Omega(\kappa)$ (and vice versa). 
Suppose $\zeta \in \partial U$. We will say that a sub-region $\Omega$ of $U$ has order of contact at most (resp. at least) $\kappa$ (with the unit circle) at the point $\zeta$ if there is a neighborhood $\Delta$ of $\zeta$ such that $\Omega \cap \Delta \subset[\zeta \Omega(\kappa)] \cap \Delta$ (resp. $\Omega \cap \Delta \supset[\zeta \Omega(\kappa)] \cap \Delta]$. We say $\Omega$ has finite order of contact at $\zeta$ if it has order of contact at most $A|\theta|^{\alpha}$ there, for some positive numbers $A$ and $\alpha$. For example, any internally tangent subdisc of $U$ has this property at the point of tangency, with $\alpha=2$.

Finally, we say $\Omega$ has order of contact at most $\kappa$ if it has at most that order of contact at each point of the circle. A simple compactness argument shows that this happens if and only if the closure of $\Omega$ meets $\partial U$ in a finite set, at each point of which $\Omega$ has order of contact at most $\kappa$.

In $\S 3$ we will require the following estimate for points on the boundary of a symmetric approach region that obeys a cone condition.

1.7. Lemma. Suppose $A$ is a positive constant, and $\kappa$ is an even contact function with $\kappa(\theta) \leq A|\theta|$ for $|\theta| \leq \pi$. If $z \in \partial \Omega(\kappa)$, then $1-|z| \geq \kappa\left(|1-z| / \sqrt{1+A^{2}}\right)$.

Proof. Write $z=r e^{i \theta}$. Then a straightforward calculation shows that

$$
|1-z|^{2}=(1-r)^{2}+4 r \sin ^{2} \frac{\theta}{2} \leq(1-r)^{2}+\theta^{2},
$$

from which follows

$$
|1-z| \leq|\theta| \sqrt{1+\left(\frac{1-r}{|\theta|}\right)^{2}} .
$$

Since additionally $z=r e^{i \theta}$ lies on the boundary of $\Omega(\kappa)$, we have $1-r=\kappa(\theta) \leq A|\theta|$, so inequality (2) yields:

$$
|1-z| \leq|\theta| \sqrt{1+A^{2}}
$$

whereupon the monotonicity and evenness of $\kappa$ imply

$$
\kappa\left(\frac{|1-z|}{\sqrt{1+A^{2}}}\right) \leq \kappa(|\theta|)=\kappa(\theta)=1-|z| .
$$

1.8. Boundary behavior of Riemann mapping functions. Carathéodory proved that every univalent (holomorphic) map taking the unit disc onto a domain $\Omega$ bounded by a Jordan curve (a Jordan domain) extends continuously to a homeomorphism (henceforth called 
the Carathéodory extension of $\varphi$ ) that takes $\bar{U}$ onto $\bar{\Omega}$ (see [22], Section 14.20, page 311). Some extra control over the derivative of $\varphi$ is provided by the following special case of a result of Warchawski. If a univalent map $\varphi$ takes $U$ onto a domain bounded by a $C^{2}$ Jordan curve, then $\varphi^{\prime}$ extends continuously to $\bar{U}$, and the extension vanishes at no point of $\bar{U}$ ([21], Theorem 10.2, page 298). To state the conclusion more succinctly: $\log \varphi^{\prime}$ extends continuously to $\bar{U}$.

These results tell us that every univalent mapping $\varphi$ of the unit disc onto an approach region $\Omega(\kappa)$ extends to a homeomorphism of the closed disc onto the closure of $\Omega(\kappa)$. If, moreover, $\kappa$ is twice continuously differentiable, then $\log \varphi^{\prime}$ extends continuously to the closed unit disc.

The $C^{2}$ requirement on $\kappa$ is not the weakest one that guarantees such extendability. The theorem of Warchawski actually asserts that something a bit weaker than $C^{1+\varepsilon}$ will suffice. However it is not enough to simply require that $\kappa$ (and therefore the boundary of $\Omega$ ) be of class $C^{1}$. A theorem of Denjoy ([21], Theorem 10.1, page 295) asserts that the $C^{1}$ condition is equivalent to the continuity of $\arg \varphi^{\prime}$ on the closed unit disc, but not, as examples show, to the continuity of $\log \varphi^{\prime}$ ([21], page 301, problem 2).

In $\S \S 3.5$ and 5.2 we will need to know how the (possible) compactness of a composition operator is influenced by the angular derivative of its inducing function.

1.9. The angular derivative. We say $\varphi$ has a (finite) angular derivative at $\zeta \in \partial U$ if there is a point $\omega \in \partial U$ such that the limit

$$
\varphi^{\prime}(\zeta) \stackrel{\text { def }}{=} \lim \frac{\omega-\varphi(z)}{\zeta-z} \quad(z \rightarrow \zeta \text { non-tangentially })
$$

exists as a finite complex number. When this happens, we call $\varphi^{\prime}(\zeta)$ the angular derivative of $\varphi$ at $\zeta$. In this case the point $\omega$ is the non-tangential limit of $\varphi$ at $\zeta$, so:

The existence of the angular derivative at a boundary point $\zeta$ implies that $\varphi$ has a non-tangential limit of modulus 1 there.

Part of the Julia-Carathéodory Theorem ([5], Sec. 298, Theorem 2.1) connects the notion of angular derivative with the limiting 
behavior of the complex derivative:

The angular derivative of $\varphi$ exists at $\zeta$ if and only if $\varphi$ has a radial limit of modulus 1 at $\zeta$, and the complex derivative of $\varphi$ has a finite nontangential limit (necessarily equal to $\left.\varphi^{\prime}(\zeta)\right)$ there.

1.10. Angular derivative and compactness. The connection between angular derivatives and compact composition operators was first noticed by the first author and P. D. Taylor ([24], Theorem 2.1): If $\varphi$ has an angular derivative at some point of $\partial U$, then the induced composition operator $C_{\varphi}$ is not compact on $H^{2}$. That is, nonexistence of the angular derivative (at each point of $\partial U$ ) is necessary for compactness. While this criterion is not sufficient for the compactness of composition operators induced by general holomorphic self-maps $\varphi$ (see [18], section 3.6) it does suffice for a large class of maps with "restricted valence" (see [18], Theorem 5.3: or [25], Corollary 3.6). In particular:

$\checkmark$ If $\varphi$ is univalent, then $C_{\varphi}$ is compact if and only if the angular derivative of $\varphi$ exists at no point of the unit circle.

2. Primer on isolation. In this section we record some preliminary results which introduce the phenomenon of isolation, and suggest that it warrants further study. We begin with a subordination result, which reinforces the connection between isolation and geometry, and then show that no compact composition operator can be isolated. In contrast, we present a new proof of Berkson's theorem, which asserts that the most strongly non-compact composition operators are isolated.

2.1. Comparison lemma. (a) Suppose $\varphi$ induces an isolated composition operator, and $\psi$ is univalent, with $\psi(U) \supset \varphi(U)$. Then $\psi$ induces an isolated composition operator.

(b) Suppose $\varphi$ is univalent, and induces a non-isolated composition operator. Then any other map $\psi$ whose image is contained in that of $\varphi$ also induces a nonisolated composition operator.

Proof. Both parts follow quickly from Littlewood's Subordination Principle. In part (a) we are assuming that there is a positive number $\delta$ such that, as measured by the operator norm, $C_{\varphi}$ lies at least $\delta$ units away from any other composition operator. The hypotheses on 
$\psi$ insure that $\varphi=\psi \circ \omega$, where $\omega=\psi^{-1} \circ \varphi$ is a holomorphic selfmap of $U$. Thus if $\chi$ is a holomorphic self-map of $U$, different from $\psi$, then $\chi \circ \omega$ is different from $\varphi$, so

$$
\begin{aligned}
\delta & \leq\left\|C_{\varphi}-C_{\chi \circ \omega}\right\|=\left\|C_{\psi \circ \omega}-C_{\chi \circ \omega}\right\|=\left\|C_{\omega}\left(C_{\psi}-C_{\chi}\right)\right\| \\
& \leq\left\|C_{\omega}\right\|\left\|C_{\psi}-C_{\chi}\right\| ;
\end{aligned}
$$

hence $C_{\psi}$ lies at least $\delta /\left\|C_{\omega}\right\|$ units distant from every other composition operator.

Part (b) follows from a similar argument, which we leave to the reader.

The next result explains why the isolation problem focuses on noncompact operators.

2.2. Proposition. The collection of compact composition operators on $H^{2}$ is arcwise connected.

Proof. For $0 \leq t \leq 1$ let $\delta_{t}$ denote the composition operator induced by the dilation $z \rightarrow t z \quad(z \in U)$. In particular: $\delta_{t}$ is compact for $0 \leq t<1, \delta_{0}$ is the "evaluation operator" that takes the function $f$ to the constant function $f(0)$, and $\delta_{1}$ is the identity operator. Now suppose $C_{\varphi}$ is compact, so the set

$$
K=C_{\varphi}\left\{\text { unit ball of } H^{2}\right\}
$$

is relatively compact in $H^{2}$. Suppose $0 \leq s \leq 1$. As $t \rightarrow s$, the operators $\delta_{t}$ converge pointwise on $H^{2}$ to $\delta_{s}$. Since these operators are all contractions, and hence equicontinuous on bounded subsets of $H^{2}$, the operators $\delta_{t}$ converge uniformly on $K$ to $\delta_{s}$. Introducing the notation $\varphi_{t}=\delta_{t} \varphi$, the last sentence asserts that the operators $C_{\varphi_{t}}=\delta_{t} C_{\varphi}$ converge uniformly on the unit ball of $H^{2}$ (i.e. in norm) to $C_{\varphi_{s}}$. Thus the mapping

$$
t \rightarrow C_{\varphi_{t}} \quad(0 \leq t \leq 1)
$$

takes the interval $[0,1]$ continuously into $\operatorname{Comp}\left(H^{2}\right)$, and therefore defines an arc of compact composition operators connecting $C_{\varphi}$ with $C_{\varphi_{0}}$, the latter being the rank one composition operator that takes a function $f$ to the constant function $f(\varphi(0))$. Since the operator norm induces on the totality of these rank one "evaluation operators" the topology (though not the metric) of the unit disc, each can be joined to the others by an arc. Thus any two compact composition operators 
can be joined by an arc that consists entirely of compact composition operators.

We close this section with our proof of Berkson's theorem. In fact we prove something more general. For each holomorphic self-map $\varphi$ of $U$, let

$$
E(\varphi)=\{\zeta \in \partial U:|\varphi(\zeta)|=1\},
$$

where we remind the reader that $\varphi(\zeta)$ denotes the radial limit of $\varphi$ at the boundary point $\zeta$. Thus $E(\varphi)$ is the set of points of the unit circle where $\varphi$ has a radial limit of modulus 1 .

2.3. TheOREM. Suppose $\varphi_{1}, \varphi_{2}, \ldots, \varphi_{n}$ are distinct holomorphic self-maps of $U$, and $a_{1}, a_{2}, \ldots, a_{n}$ are complex scalars. Then

$$
\left\|\sum_{j=1}^{n} a_{j} C_{\varphi_{j}}\right\|^{2} \geq \sum_{j=1}^{n}\left|a_{j}\right|^{2} \sigma\left(E\left(\sigma_{j}\right)\right) .
$$

2.4. Corollary (Cf. Berkson [2]). If $\varphi \neq \psi$, then

$$
\left\|C_{\varphi}-C_{\psi}\right\| \geq \sqrt{\sigma(E(\varphi))+\sigma(E(\psi))} .
$$

Note the following consequences of Corollary 2.4.: (i) if $\sigma(E(\varphi))>$ 0 , then $C_{\varphi}$ is isolated, in the operator norm topology, from every other composition operator, and even (by Theorem 2.3) from any linear combination of composition operators not induced by $\varphi$.

(ii) Every inner function lies at least one norm unit distant from every other composition operator (as we pointed out in the Introduction, this was first noticed by Berkson, with $1 / \sqrt{2}$ instead of 1 ), and at least $\sqrt{2}$ units distant from any other inner function.

Proof of Theorem 2.3. For ease of notation, we write

$$
T=\sum_{j=1}^{n} a_{j} C_{\varphi_{j}}
$$

and let an unadorned integral sign $\int$ mean $\int_{\partial U}$. For $\alpha \in U$, let

$$
f_{\alpha}=k_{\alpha} /\left\|k_{\alpha}\right\| \text {, }
$$

denote the "normalized reproducing kernel" for the point $\alpha$. Thus $\left\|f_{\alpha}\right\|=1$, and

$$
f_{\alpha}(z)=\frac{\sqrt{1-|\alpha|^{2}}}{1-\bar{\alpha} z} \quad(z \in U)
$$


Thus:

$$
\begin{aligned}
\|T\|^{2} & \geq \limsup _{|r| \rightarrow 1-} \sup _{|\zeta|=1}\left\|T\left(f_{r \zeta}\right)\right\|^{2} \\
& \geq \limsup _{|r| \rightarrow 1-} \int\left\|T\left(f_{r \zeta}\right)\right\|^{2} d \sigma(\zeta)
\end{aligned}
$$

Upon expanding the integrand on the right, using the definition of $T$ and the expression 1.2(3) for the inner product as a boundary integral, we obtain

$$
\|T\|^{2} \geq \limsup _{|r| \rightarrow 1-} \sum_{i, j=1}^{n} a_{i} \bar{a}_{j} \int Q_{i j}(r \zeta) d \sigma(\zeta),
$$

where for each $0 \leq r<1$ and $\zeta \in \partial U$ :

$$
\begin{aligned}
& Q_{i j}(r \zeta) \stackrel{\text { def }}{=}\left\langle C_{\varphi_{l}} f_{r \zeta}, C_{\varphi_{j}} f_{r \zeta}\right\rangle \\
& =\left(1-r^{2}\right)\left\langle k_{r \zeta} \circ \varphi_{i}, k_{r \zeta} \circ \varphi_{j}\right\rangle \\
& =\int \frac{1-r^{2}}{\left(1-r \bar{\zeta} \varphi_{i}(\eta)\right)\left(1-r \overline{\varphi_{j}(\eta)}\right)} d \sigma(\eta) \\
& =\int\left(1-r^{2}\right) \overline{k_{r \varphi_{i}(\eta)}(\zeta)} k_{r \varphi_{j}(\eta)}(\zeta) d \sigma(\eta) .
\end{aligned}
$$

Now integrate both sides of the above equation over $\partial U$, and apply Fubini's Theorem:

$$
\begin{aligned}
\int Q_{i j}(r \zeta) d \sigma(\zeta) & =\int\left(1-r^{2}\right)\left\langle k_{r \varphi_{j}(\eta)}, k_{r \varphi_{i}(\eta)}\right\rangle d \sigma(\eta) \\
& =\int\left(1-r^{2}\right) k_{r \varphi_{j}(\eta)}\left(r \varphi_{i}(\eta)\right) d \sigma(\eta) \quad[\text { by 1.3(1)] } \\
& =\int \frac{1-r^{2}}{1-r^{2} \varphi_{i}(\eta) \overline{\varphi_{j}(\eta)}} d \sigma(\eta) .
\end{aligned}
$$

The integrand in the last line is bounded by 1 , and as $r \rightarrow 1-$ it converges to the characteristic function of the set

$$
E_{i j} \stackrel{\text { def }}{=}\left\{\eta \in \partial U: \varphi_{i}(\eta) \overline{\varphi_{j}(\eta)}=1\right\} .
$$

Thus the Lebesgue Dominated Convergence Theorem yields

$$
\lim _{r \rightarrow 1-} \int Q_{i j}(r \zeta) d \sigma(\zeta)=\sigma\left(E_{i j}\right),
$$

so by inequality $(*)$ above,

$$
\|T\|^{2} \geq \sum_{i, j=1}^{n} a_{i} \bar{a}_{j} \sigma\left(E_{i j}\right)
$$


If $i=j$, then $E_{i j}$ is just $E\left(\varphi_{j}\right)$. On the other hand, if $i \neq j$, then $\varphi_{i} \neq \varphi_{j}$, and since the radial limit functions of two different bounded holomorphic functions cannot coincide on a subset of positive measure in $\partial U$ (by the log-integrability result mentioned in $\S 1.4$; or [22], Theorem $17.10(\mathrm{c})$, page 366), we must have $\sigma\left(E_{i j}\right)=0$. These observations, along with the last inequality, complete the proof of the theorem.

This proof employed a very weak consequence of the fact that the boundary function of a non-trivial bounded holomorphic function is log-integrable. In $\S 4$ we use the full strength of this log-integrability to produce isolation in a much more delicate setting.

3. Non-isolation. Berkson's theorem asserts that certain very strongly extreme points in the unit ball of $H^{\infty}$ induce isolated composition operators. The main result of this section says that isolated composition operators can only be induced by extreme points. We state it, however, in more utilitarian form.

3.1. THEOREM ("Isolated $\Rightarrow$ Extreme"). If $\varphi$ is a holomorphic selfmap of $U$ for which

$$
\int_{\partial U} \log (1-|\varphi|) d \sigma>-\infty
$$

then $C_{\varphi}$ is not isolated in $\operatorname{Comp}\left(H^{2}\right)$.

At the end of the section we will show that condition $(*)$ fails to characterize the nonisolated composition operators. We will also observe that a (rather strong) sufficient condition for $(*)$ to hold is that $\varphi(U)$ have finite order of contact with the unit circle. Thus, for example, the composition operator induced by the mapping $\varphi(z)=$ $(1+z) / 2$, shown by Schwartz to be non-compact, is not isolated.

It is instructive to see why the question of isolation is not completely trivial for this particular operator. Certainly there is an obvious candidate for a family of composition operators with a cluster point at $C_{(1+z) / 2}$, namely the ones induced by the maps

$$
\varphi_{t}(z)=(1-t)+t z \quad(0<t<1) .
$$

However, upon applying these composition operators to the common eigenfunctions

$$
f_{\alpha}(z)=(1-z)^{\alpha} \quad(\operatorname{Re} \alpha>-1 / 2),
$$


which are easily seen to belong to $H^{2}$ for the indicated range of $\alpha$, we obtain the inequality

$$
\left\|C_{\varphi_{s}}-C_{\varphi_{t}}\right\| \geq\left|s^{\alpha}-t^{\alpha}\right| \quad(0<s, t<1, \operatorname{Re} \alpha>-1 / 2),
$$

the right side of which, for $s$ and $t$ fixed, and $s \neq t$, tends to $s^{-1 / 2}+$ $t^{-1 / 2}$ when $\alpha$ tends appropriately to the line $\operatorname{Re} z=-1 / 2$. Thus we obtain the lower bound

$$
\left\|C_{\varphi_{s}}-C_{\varphi_{t}}\right\| \geq \frac{1}{\sqrt{s}}+\frac{1}{\sqrt{t}} \text { for } 0<s, t<1,
$$

which asserts that, far from clustering anywhere at all, the operators in question form a discrete set in the operator norm.

The proof of Theorem 3.1 hinges on the following estimate for the norm of a difference of two composition operators.

3.2. Difference Theorem. For each pair $\varphi, \psi$ of distinct holomorphic self-maps of $U$, define

$$
I(\varphi, \psi)=\int \frac{|\varphi-\psi|^{2}}{(\min \{1-|\varphi|, 1-|\psi|\})^{3}} d \sigma .
$$

If $I(\varphi, \psi)<\infty$, then $\left\|C_{\varphi}-C_{\psi}\right\| \leq \sqrt{I(\varphi, \psi)}$, and in addition, $C_{\varphi}-C_{\psi}$ is compact.

Proof. Since $\varphi$ and $\psi$ are distinct, their radial limit functions coincide on at most a boundary set of measure zero, so the convergence of the integral $I(\varphi, \psi)$ implies that both functions have modulus $<1$ at almost every point of the unit circle. Suppose $\zeta$ is such a point, and set $\alpha=\varphi(\zeta)$ and $\beta=\psi(\zeta)$ : both points of $U$. Let $\Gamma$ denote the line segment joining these points. Then:

$$
\begin{aligned}
\mid C_{\varphi} & f(\zeta)-C_{\psi} f(\zeta)|=| f(\alpha)-f(\beta) \mid \\
& =\left|\int_{\Gamma} f^{\prime}(\zeta) d \zeta\right| \leq \int_{\Gamma}\left|f^{\prime}(\zeta)\right||d \zeta| \\
& \leq\|f\| \int_{\Gamma} \frac{|d \zeta|}{(1-|\zeta|)^{3 / 2}} \quad[\text { by 1.3(4)], } \\
& \leq\|f\| \frac{|\alpha-\beta|}{(\min \{1-|\alpha|, 1-|\beta|\})^{3 / 2}},
\end{aligned}
$$

where the final step uses the fact that $\Gamma$ is the line segment joining $\alpha$ 
and $\beta$. Summarizing:

(1) $\left|C_{\varphi} f(\zeta)-C_{\psi} f(\zeta)\right| \leq\|f\| \frac{|\varphi(\zeta)-\psi(\zeta)|}{(\min \{1-|\varphi(\zeta)|, 1-|\psi(\zeta)|\})^{3 / 2}}$

(a.e. $\zeta \in \partial U$ ). The desired norm estimate follows upon squaring both sides of this inequality, integrating over $\partial U$, and using expression $1.2(2)$ for the $H^{2}$ norm as a boundary integral.

Involved in the norm computation of the last sentence is the tacit assumption that the radial limit of the holomorphic function $f \circ \varphi$ coincides a.e. with the composition of the corresponding radial limits. While not completely obvious, this follows from an argument involving Lindelöf's theorem, or a polynomial approximation argument ([22], Lemma 3, page 44). Better still, one can get around the problem completely by assuming that the function $f$ in the estimates above is a holomorphic polynomial. This involves no loss of generality, because these polynomials are dense in $H^{2}$.

We next show that the finiteness of the integral $I(\varphi, \psi)$ implies the compactness of the operator difference $C_{\varphi}-C_{\psi}$. Since this fact will not be needed until $\S 5$, the reader may wish to temporarily (perhaps permanently!) skip its proof. Suppose $\left\{f_{n}\right\}$ is a sequence in $H^{2}$ that converges weakly to zero. We must show that $\left\|\left(C_{\varphi}-C_{\psi}\right) f_{n}\right\| \rightarrow 0$. Weakly convergent sequences are bounded, so we may without loss of generality suppose that all the functions $f_{n}$ lie in the unit ball of $H^{2}$. Finally, note that thanks to inequality $1.3(3)$, the sequence $\left\{f_{n}\right\}$ converges to zero uniformly on compact subsets of $U$.

Let $\varepsilon>0$ be given. Since the integral $I(\varphi, \psi)$ converges, and the boundary functions of $\varphi$ and $\psi$ exist and have modulus $<1$ everywhere off an exceptional set $E$ (possibly the empty set) of measure zero, there is an open subset $V$ of $\partial U$, containing $E$, such that

$$
|\varphi(\zeta)|>1-\varepsilon \text { and }|\psi(\zeta)|>1-\varepsilon \quad(\text { a.e. } \zeta \in V),
$$

and

$$
\int_{V} \frac{|\varphi-\psi|^{2}}{(\min \{1-|\varphi|, 1-|\psi|\})^{3}} d \sigma<\frac{\varepsilon^{2}}{2}
$$

By (2) above, and the definition of $V$, both sets $\varphi(\partial U \backslash V)$ and $\psi(\partial U \backslash V)$ lie in a compact subset of $U$, so the sequence $\left\{f_{n}\right\}$ converges uniformly to zero on both. This provides a positive integer $N$ so that whenever $n>N$

$$
\left|f_{n} \circ \varphi-f_{n} \circ \psi\right|<\varepsilon^{2} / 2 \text { on } \partial U \backslash V .
$$


Thus by (1) and (2)

$$
\begin{aligned}
\left\|\left(C_{\varphi}-C_{\psi}\right) f_{n}\right\|^{2} & =\int\left|f_{n}(\varphi(\zeta))-f_{n}(\psi(\zeta))\right|^{2} d \sigma(\zeta) \\
& =\int_{V}+\int_{\partial U \backslash V}\left|f_{n}(\varphi(\zeta))-f_{n}(\psi(\zeta))\right|^{2} d \sigma(\zeta)
\end{aligned}
$$

[by (1) and (4)] $\leq\left\|f_{n}\right\|^{2} \int_{V} \frac{|\varphi-\psi|^{2}}{(\min \{1-|\varphi|, 1-|\psi|\})^{3}} d \sigma+\frac{\varepsilon^{2}}{2}$

[by $(3)]<\left\|f_{n}\right\|^{2} \frac{\varepsilon^{2}}{2}+\frac{\varepsilon^{2}}{2} \leq \frac{\varepsilon^{2}}{2}+\frac{\varepsilon^{2}}{2}=\varepsilon^{2}$,

where in the last line we finally use the fact that the entire sequence $\left\{f_{n}\right\}$ is assumed to lie in the unit ball of $H^{2}$. Thus we have the desired result $\left\|\left(C_{\varphi}-C_{\psi}\right) f_{n}\right\| \rightarrow 0$; hence the operator $C_{\varphi}-C_{\psi}$ is compact on $H^{2}$.

Proof of Theorem 3.1. The hypothesis $(*)$ of the theorem insures that there is an outer function $\omega$ on $U$ such that

$$
|\omega|=(1-|\varphi|)^{3 / 2} \quad \text { a.e. on } \partial U
$$

(§1.4). Since the modulus of $\omega$ is bounded by one a.e. on $\partial U$, we have $|\omega(z)|<1$ for each $z \in U(\S 1.5)$, and hence the holomorphic function

$$
\varphi_{t} \stackrel{\text { def }}{=} \varphi+t \omega
$$

is bounded on $U$ for every real $t$.

We claim that $\varphi_{t}(U) \subset U$ whenever $|t|<1$. To see this, observe that a.e. on $\partial U$ we have

$$
|\omega|=(1-|\varphi|)^{3 / 2} \leq 1-|\varphi|,
$$

so

$$
\left|\varphi_{t}\right| \leq|\varphi|+|t||\omega| \leq|\varphi|+(1-|\varphi|) \leq 1
$$

Thus $\left|\varphi_{t}\right|<1$ at every point of $U$, which proves the claim.

We are going to prove that $C_{\varphi}$ is not isolated by using the Difference Theorem to show that $\left\|C_{\varphi}-C_{\varphi_{t}}\right\| \rightarrow 0$ as $t \rightarrow 0$. From the definitions we see that a.e. on $\partial U$ :

$$
1-\left|\varphi_{t}\right|=1-|\varphi+t \omega| \geq(1-|\varphi|)-|t||\omega|=|\omega|^{2 / 3}-|t||\omega|
$$

$$
1-\left|\varphi_{t}\right| \geq(1-|t|)|\omega|^{2 / 3} \quad \text { a.e. on } \partial U
$$


where the last inequality follows from the above-mentioned fact that $|\omega| \leq 1$ a.e. on $\partial U$.

By (1) and (2) the integrand in the definition of $I\left(\varphi, \varphi_{t}\right)$ has denominator $\geq(1-|t|)^{3}|\omega|^{2}$, and numerator $=|t|^{2}|\omega|^{2}$ a.e. on $\partial U$. Thus the integrand, and therefore $I\left(\varphi, \varphi_{t}\right)$ itself, is bounded by the constant $|t|^{2} /(1-|t|)^{3}$. By the Difference Theorem,

$$
\left\|C_{\varphi}-C_{\varphi_{t}}\right\| \leq|t| /(1-|t|)^{3 / 2} \rightarrow 0 \text { as } t \rightarrow 0,
$$

so the theorem is proved.

As an application of Theorem 3.1, we have the following result, which shows, as a special case, that $C_{\varphi}$ is not isolated whenever $\varphi(U)$ has at most finite order contact with the unit circle. In fact, even limited "exponential order" contact is allowed.

3.3. CoRollary. Suppose $\varphi(U)$ has order of contact at most $\kappa$ with $\partial U$, where

$$
\kappa(\theta)=\exp \left(-B|\theta|^{-\alpha}\right) \quad(|\theta| \leq \pi)
$$

for some constants $B>0$, and $0<\alpha<1$. Then $C_{\varphi}$ is not isolated in $\operatorname{Comp}\left(H^{2}\right)$.

Proof. The hypothesis states that there are finitely many points $\eta_{j}$, $(1 \leq j \leq n)$ on the unit circle, such that for each index $j$, there is an open disc $\Delta_{j}$, centered at $\eta_{j}$, with $\Omega \cap \Delta_{j} \subset \eta_{j} \Omega(\kappa) \quad(1 \leq j \leq n)$, where $\kappa(\theta)=\exp \left(-B /|\theta|^{\alpha}\right)$. We may of course choose these discs to be pairwise disjoint.

By Lemma 2.1 (the "Comparison Lemma"), we may also assume that in each $\Delta_{j}$ the image of $\varphi$ is a Jordan region $\Omega$ whose boundary coincides with that of the approach region $\eta_{j} \Omega(\kappa)$, and that $\varphi$ is a univalent mapping taking $U$ onto $\Omega$. As is our custom, we also use " $\varphi$ " to denote the Carathéodory extension of this map to a homeomorphism of $\bar{U}$ onto $\bar{\Omega}(\S 1.8)$.

For $1 \leq j \leq n$, the set

$$
I_{j}=\varphi^{-1}\left(\partial \varphi(U) \cap \Delta_{j}\right)
$$

is an arc of the unit circle, at each point $\zeta$ of which we have arranged that

$$
1-|\varphi(\zeta)|=\kappa\left(\arg \varphi(\zeta)-\arg \eta_{j}\right)
$$


Since our contact function $\kappa$ obeys the hypotheses of Lemma 1.7, equation (1) implies that (upon possibly enlarging the constant $B$ )

$$
1-|\varphi(\zeta)| \geq \exp \left(\frac{-B}{\left|\varphi(\zeta)-\eta_{j}\right|^{\alpha}}\right) \quad\left(z \in I_{j}, \quad 1 \leq j \leq n\right) .
$$

Because $\alpha<1$, each function

$$
f_{j}(z)=\left(\zeta_{j}-z\right)^{-\alpha / 2} \quad(z \in U)
$$

belongs to $H^{2}$; hence by Littlewood's Principle, so does $C_{\varphi} f_{j}$. In other words, for each index $j$, the logarithm of the right side of (2) is integrable over the unit circle. Since $|\varphi|$ is bounded away from 1 on $\partial U \backslash \bigcup_{j} I_{j}$, this shows that $\log (1-|\varphi|)$ is integrable over $\partial U$. This observation, along with Theorem 3.2, completes the proof.

3.4. Remarks. (a) The proof of Theorem 3.1, repeated with just a little more care, shows that

$$
\lim _{s \rightarrow t}\left\|C_{\varphi_{s}}-C_{\varphi_{t}}\right\| \rightarrow 0 \quad(|t|<1),
$$

i.e., that the map $t \rightarrow C_{\varphi_{t}}$ takes the interval $(-1,1)$ continuously into $\operatorname{Comp}\left(H^{2}\right)$. Thus:

$\checkmark$ If $\varphi$ is not an extreme point, then $C_{\varphi}$ lies in an arc in $\operatorname{Comp}\left(H^{2}\right)$.

The Difference Theorem also makes a statement about compactness, which in this context asserts that: each member of the arc mentioned above has compact difference with every other member.

(b) The parameters $s$ and $t$ occurring in the remark above could as well have been complex numbers, or even bounded holomorphic functions on $U$. For complex numbers, the proof of Theorem 3.1, improved according to remark (a), asserts that the map $t \rightarrow C_{\varphi_{t}}$ takes the unit disc continuously into $\operatorname{Comp}\left(H^{2}\right)$, while for holomorphic functions it shows that the same thing happens to the unit ball of $H^{\infty}$.

(c) The exponent $\alpha$ in Corollary 3.3 cannot be taken to be 1 . The main result of the next section shows, for example, that the Riemann mapping function taking $U$ onto the domain $\Omega(\kappa)$, where $\kappa(\theta)=$ $\exp (-B /|\theta|)$, induces an isolated composition operator on $H^{2}$.

Theorem 3.1 asserts that "isolated implies extreme." We close this section by showing that the converse is not true, even for composition operators induced by univalent maps. 
3.5. THEOREM ("Extreme, yet Compact"). There exists a univalent, holomorphic self-map $\varphi$ of $U$ such that

$$
\int_{\partial U} \log (1-|\varphi|) d \sigma=-\infty,
$$

yet $C_{\varphi}$ is compact on $H^{2}$, and therefore not isolated in $\operatorname{Comp}\left(H^{2}\right)$.

Proof. We begin with an approach region $\Omega(\kappa)$, where $\kappa$ is any even $C^{2}$ contact function with

$$
\kappa(\theta)=e^{-1 /|\theta|} \quad(0<|\theta| \leq \pi / 2),
$$

and $\kappa(0)=0$. Since $\Omega(\kappa)$ is symmetric about the real axis, there is a univalent mapping $\varphi_{\kappa}$ of $U$ onto $\Omega(\kappa)$ that takes the top half of $U$ onto the top half of $\Omega(\kappa)$, and, of course, treats bottom halves similarly. By the discussion of $\S 1.8$, the Carathéodory extension (also denoted by $\varphi_{\kappa}$ ) of this map provides a $C^{1}$ homeomorphism of $\bar{U}$ onto $\bar{\Omega}(\kappa)$, which necessarily fixes the "vertex" 1 , and whose derivative never vanishes on $\bar{U}$.

Although not required for the proof, a routine argument involving boundary regularity shows that $\varphi_{\kappa}$ is an extreme point, and the results of the next section will show that its induced composition operator is isolated. The goal of the rest of this section is to show that $\varphi_{\kappa}$ can be modified so that the extreme point property is preserved, but isolation is not.

The desired adjustment is effected by a univalent map $T$ that takes $U$ onto $U^{+}$. It is the map $\varphi=T \circ \varphi_{\kappa}$ that we will show has the required properties.

An explicit representation for $T$ involves the Möbius transformation

$$
\tau(z)=i \frac{1-z}{1+z} \quad(z \in \bar{U}),
$$

which takes $U$ conformally onto $\Pi^{+}$, the top half of $U$ onto the (open) first quadrant, and the unit circle onto the real line. Define

$$
T(z)=\tau^{-1} \sqrt{\tau(z)} \quad(z \in \bar{U}),
$$

where the square root is defined on the complex plane cut along the negative real axis, and takes values in the upper half plane. The mapping $T$ fixes the points $-1, i$, and 1 , while sending the point $-i$ to 0 . Moreover, the derivative $T^{\prime}(z)$ tends to $\infty$ (uniformly) as $z$ tends to 1 through $U$. By the chain rule and the fact that $\varphi_{\kappa}^{\prime}$ is bounded away from zero on $U$, the function $\varphi^{\prime}(z)$ also tends to infinity as $z$ 
tends to 1 . Thus by the discussion of $\S \S 1.9$ and 1.10 , relating angular derivatives to compactness, and by the fact that $\varphi$ is univalent, the operator $C_{\varphi}$ is compact on $H^{2}$.

To see that $\log (1-|\varphi|)$ is not integrable, we need two elementary estimates whose derivation we leave to the reader.

$$
1-\left|\tau^{-1}(w)\right|^{2}=\frac{4 \operatorname{Im} w}{|1-i w|^{2}}<4 \operatorname{Im} w \quad\left(w \in \Pi^{+}\right) .
$$

$$
\operatorname{Im} \sqrt{w}<\sqrt{\frac{\operatorname{Im} w}{2}} \quad\left(w \in \Pi^{+}, \operatorname{Re} w>0\right) .
$$

Because $\tau$ maps the top half of the unit disc into the first quadrant, these inequalities yield:

$$
1-|T(z)|<4 \sqrt{1-|z|} \quad\left(z \in U^{+}, \operatorname{Re} z>0\right) .
$$

Now the arc

$$
\Gamma=\varphi_{\kappa}^{-1}\left\{\partial \Omega(\kappa) \cap \text { first quadrant } \cap \text { exterior of } \frac{1}{4} U\right\},
$$

lies on the top half of the unit circle and has 1 as its "right hand endpoint." Fix $\zeta \in \Gamma$, and write $\varphi_{\kappa}(\zeta)=r e^{i \theta}$, with $|\theta|<\pi$. Since $\varphi_{\kappa}(\zeta)$ is in the first quadrant, (3) implies that

$$
1-|\varphi(\zeta)|=1-\left|T\left(\varphi_{\kappa}(\zeta)\right)\right|<4 \sqrt{1-\left|\varphi_{\kappa}(\zeta)\right|} .
$$

Since also $\varphi_{\kappa}(\zeta) \in \partial \Omega(\kappa)$, we have

$$
1-\left|\varphi_{\kappa}(\zeta)\right|=\kappa(\theta) \leq \kappa\left(\frac{\pi}{4}\left|1-\varphi_{\kappa}(\zeta)\right|\right)=\exp \left\{\frac{-\pi}{4\left|1-\varphi_{\kappa}(\zeta)\right|}\right\},
$$

where the inequality follows from the monotonicity of $\kappa$, and a little manipulation with the identity in line (1) of the proof of Lemma 1.7, which uses the fact that $r=\left|\varphi_{\kappa}(\zeta)\right| \geq \frac{1}{4}$. The last two displayed inequalities yield:

$$
1-|\varphi(\zeta)| \leq 4 \exp \left\{\frac{-\pi}{8\left|1-\varphi_{\kappa}(\zeta)\right|}\right\} \quad(\zeta \in \Gamma)
$$

Since the derivative of $\varphi_{\kappa}$ extends continuously to $\bar{U}$, and $\varphi_{\kappa}(1)=1$, we have

$$
\left|1-\varphi_{\kappa}(\zeta)\right| \leq C|1-\zeta| \quad(\zeta \in \partial U),
$$

where $C$ is the supremum of $\left|\varphi_{\kappa}^{\prime}(z)\right|$ over the unit disc. By the last two inequalities,

$$
\log (1-|\varphi(\zeta)|) \leq \log 4-\frac{\pi}{8 C|1-\zeta|} \quad(\zeta \in \Gamma)
$$


Since $\Gamma$ is an arc of the unit circle having 1 as an endpoint, the function $|1-\zeta|^{-1}$ is not integrable over it, and so, by the last inequality, neither is $\log (1-|\varphi(\zeta)|)$.

4. Isolation and contact. In this section we offer a partial converse to Theorem 3.1 ("isolated implies extreme"), by showing that if $\varphi$ is a sufficiently regular univalent extreme point, then the induced composition operator is isolated in $\operatorname{Comp}\left(H^{2}\right)$. The additional regularity is phrased in terms of the notions of "contact function" and "order of contact" that were introduced in $\S 1.6$. Here is the main result of the section.

4.1. THEOREM. Suppose $\kappa$ is a $C^{2}$ contact function, and $\varphi$ is a holomorphic, univalent self-map of $U$ whose image has order of contact at least $\kappa$ with $\partial U$. If

$$
\int_{0}^{\pi} \log \kappa(t) d t=-\infty
$$

then $C_{\varphi}$ is isolated in $\operatorname{Comp}\left(H^{2}\right)$.

At the end of this section (Remarks 4.11) we will justify the contention that $\varphi$ is an extreme point. Note that this theorem contains, as a special case, the example mentioned in Remark 3.4(c). Note further that the integrability requirement is "one-sided:" it is only stated for the part of $\Omega(\kappa)$ above the real axis. It could, of course, equally well be stated for the lower part of $\Omega(\kappa)$.

As we pointed out at the end of $\S 1.8$, the smoothness hypothesis on $\kappa$, which insures that Riemann mapping functions onto $\Omega(\kappa)$ have continuously differentiable Carathéodory extensions, could be weakened considerably, but not, as shown by Theorem 3.5, omitted completely.

The proof of Theorem 4.1, will occupy most of the rest of this section. For convenience we break it into a number of smaller steps.

4.2. Preliminary reductions. Thanks to Comparison Lemma 2.1, we can suppose without loss of generality that the image of $\varphi$ is an approach region $\Omega(\kappa)$, as described in $\S 1.6$, where $\kappa$ satisfies the nonintegrability condition (*) above. According to the discussion of $\S 1.8$, we may, as in the previous section, regard $\varphi$ as a $C^{1}$ homeomorphism of $\bar{U}$ onto $\bar{\Omega}(\kappa)$ that fixes the boundary point 1 , and has nonvanishing derivative on $\bar{U}$. 
For much of the proof of Theorem 4.1, we will find it convenient to transfer our attention to the upper half-plane $\Pi^{+}$. This is accomplished through the Möbius transformation $\tau$ of $\S 3.5$, which we recall takes $U$ conformally onto $\Pi^{+}$, the unit circle onto the real line, and the point 1 to the origin. Normalized Lebesgue measure on $\partial U$ is taken by this map to the measure

$$
\frac{1}{\pi} \frac{d x}{1+x^{2}}
$$

on $\mathbb{R}$. Thus if $f \in H^{2}$, and $F=f \circ \tau^{-1}$ is the corresponding function on $\Pi^{+}$, then

$$
\|f\|_{2}^{2}=\frac{1}{\pi} \int_{-\infty}^{\infty}|F(x)|^{2} \frac{d x}{1+x^{2}} .
$$

The approach region $\Omega(\kappa)$ gets carried by $\tau$ onto a Jordan region in $\Pi^{+}$, which we simply denote by $\Omega$, whose boundary, in a neighborhood of the origin has cartesian equation

$$
y=h(x) \quad(|x| \leq a \leq 1),
$$

where $h:[-a, a] \rightarrow[0, \infty)$ is a twice continuously differentiable function that vanishes precisely at the origin, and (as the reader can verify after a little calculation) is monotonically increasing on $[0, a]$. Finally, the non-integrability condition $4.1(*)$ for $\kappa$ becomes

$$
\int_{0}^{a} \log h(x) d x=-\infty
$$

As for the map $\varphi$, its counterpart is the univalent map $\Phi=\tau \circ \varphi \circ$ $\tau^{-1}$ that takes $\Pi^{+}$onto $\Omega$, and extends to a $C^{1}$ homeomorphism between the closures of these regions, with derivative bounded away from zero in a neighborhood of the origin.

4.3. Notation. We will, with occasional exceptions, adhere to the convention that upper case letters such as $\Phi, \Psi, F$, refer to holomorphic functions on $\Pi^{+}$, while the corresponding lower case letters denote their counterparts, via $\tau$, on the unit disc.

The symbol $I$, with or without subscripts, will denote an arc of the unit circle, or a finite interval of the real line. If $I \subset \mathbb{R}$, then we denote by $I / 2$ the interval with the same center as $I$, but half the length. We use absolute value signs to denote ordinary Lebesgue measure on the real line. For example, if $I$ is an interval of the line, then $|I|$ denotes its length. 
4.4. Harmonic measure of an interval. Suppose $I=[a, b]$ is a real interval. Then (already contradicting our upper vs. lower case convention) the harmonic measure of $I$ is

$$
\omega_{I}(z)=\frac{1}{\pi} \arg \frac{z-b}{z-a} \quad\left(z \in \Pi^{+}\right) .
$$

This is the unique bounded harmonic function on the upper half-plane that assumes boundary values 1 a.e. on the interval $I$, and zero a.e. off $I$.

We will need to estimate the size of harmonic measure on some important subsets of $\Pi^{+}$. Let $T(I)$ denote the open half-disc in $\Pi^{+}$ with $I$ as diameter. A little plane geometry shows that

$$
T(I)=\left\{z \in \Pi^{+}: \omega_{I}(z)>1 / 2\right\} .
$$

For $\delta>0$, define

$$
S(I, \delta)=\left\{z \in \Pi^{+}: \operatorname{Re} z \in I / 2 \text { and } \operatorname{Im} z<\delta|I| / 2\right\},
$$

the open rectangle whose base is $I / 2$, and whose height is $\delta$ times the length of its base. Thus $S(I, \delta) \subset T(I)$ for each $0<\delta<\frac{1}{2} \sqrt{3}$. We have the following crucial estimate:

\subsection{Lemma. $1-\omega_{I}(z) \leq \delta$ for all $z \in S(I, \delta)$.}

Proof. It is enough to prove the result for $I=[-1,1]$, and $\operatorname{Re} z \geq$ 0 . Fix $z=x+i y \in S(I, \delta)$, and write $\theta_{1}=\arg (z-1), \theta_{2}=$ $\arg (z+1)$. Then $\omega_{I}(z)=\left(\theta_{1}-\theta_{2}\right) / \pi$, so a look at the relevant right triangles show

$$
\begin{aligned}
1-\omega_{I}(z) & =\frac{1}{\pi}\left[\left(\pi-\theta_{1}\right)+\theta_{2}\right] \\
& =\frac{1}{\pi}\left[\arctan \frac{y}{1-x}+\arctan \frac{y}{1+x}\right] \\
& \leq \frac{1}{\pi}\left[\frac{y}{1-x}+\frac{y}{1+x}\right] \\
& =\frac{2 y}{\pi\left(1-x^{2}\right)} \leq 8 y / 3 \pi \leq \delta,
\end{aligned}
$$

where the next-to-last inequality holds because $0 \leq x \leq 1 / 2$.

Figure 1 summarizes the estimates obtained so far for harmonic measure. 


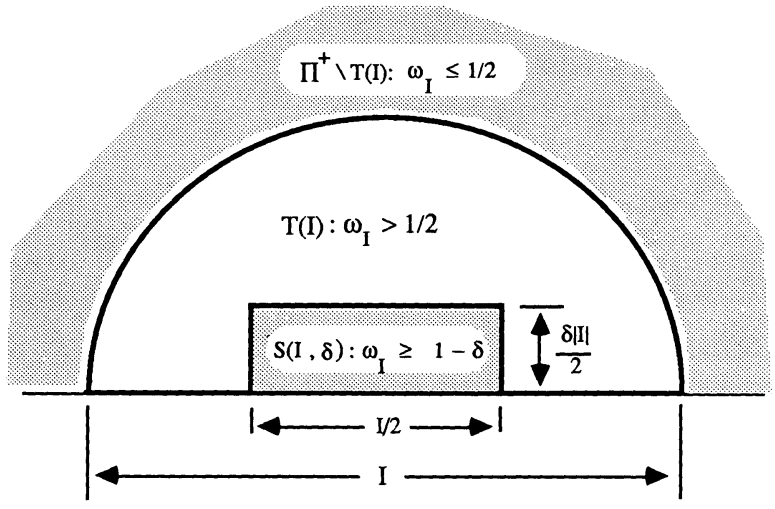

FIGURE 1. Estimates for $\omega_{I}$

4.6. Test functions. In this section we construct the class of holomorphic test functions that will be used to estimate the norms of differences of composition operators. For each finite real interval $I$, let

$$
u_{I}=2 \omega_{I}-1 \text {, }
$$

so $u_{I}$ is the bounded harmonic function on $\Pi^{+}$whose boundary function takes the value +1 a.e. on $I$, and -1 a.e. off $I$. Set

$$
F_{I}=\exp \left\{\left(-\frac{1}{2} \log |I|\right)\left(u_{I}+i \tilde{u}_{I}\right)\right\},
$$

where $\tilde{u}_{I}$ is the harmonic conjugate of $u_{I}$ in $\Pi^{+}$. Thus $F_{I}$ is a bounded holomorphic function in $\Pi^{+}$whose boundary function has modulus given by

$$
\left|F_{I}\right|^{2}= \begin{cases}\frac{1}{|I|} & \text { a.e. on } I \\ |I| & \text { a.e. off } I .\end{cases}
$$

It follows readily from this, and the discussion of $\S 4.2$, that if $f_{I}=$ $F_{I} \circ \tau$, then

$$
1 / 3 \leq\left\|f_{I}\right\| \leq 3 \text { if } I \subset[-1,1],
$$

(where the constants, though correct, are not meant to be taken too seriously). The pointwise estimates of $\omega_{I}$ summarized in Figure 1 translate into corresponding estimates of the modulus of $F_{I}$.

4.7. Pointwise estimates on $\left|F_{I}\right|$. If $|I| \leq 1$, then

(a) $\left|F_{I}\right|^{2} \leq 1$ in $\Pi^{+} \backslash T(I)$,

(b) $\left|F_{I}\right|^{2} \geq(1 /|I|) \exp \{2 \delta \log |I|\}$ in $S(I, \delta)$. 
Proof. by definition,

$$
\left|F_{I}\right|^{2}=\exp \left\{-u_{I}(z) \log |I|\right\} \quad\left(z \in \Pi_{+}\right) .
$$

We have already observed that $\omega_{I} \leq 1 / 2$ in $\Pi^{+} \backslash T(I)$, so $u_{I} \leq 0$, and therefore (since $\log |I| \leq 0) \quad\left|F_{I}\right| \leq 1$ in that set. This proves (a). Part (b) follows from Lemma 4.5, which asserts that for $z \in S(I, \delta)$ we have

$$
1-u_{I}(z)=2\left(1-\omega_{I}(z)\right) \leq 2 \delta
$$

hence

$$
u_{I}(z) \geq 1-2 \delta,
$$

which, along with (1) above yields

$$
\left|F_{I}\right|^{2} \geq \exp \left\{(1-2 \delta)(-\log |I|)=\frac{1}{|I|} \exp \{2 \delta \log |I|\}\right\},
$$

as desired.

4.8. Choosing the proper test functions. Recall that we began with a univalent map $\varphi$ taking $U$ onto an approach region, and this situation was carried over to a mapping $\Phi$ of the upper half-plane onto a region $\Omega$ whose boundary, in a neighborhood of the origin is the cartesian graph

$$
y=h(x) \quad(|x| \leq a \leq 1),
$$

where $h$ is of class $C^{2}$ on the interval $[-a, a]$, strictly positive there, except at the origin where it takes the value zero, and monotonically increasing on $(0, a]$. Recall also the crucial assumption on the degree of contact that $\Omega$ has with the positive real axis:

$$
\int_{0}^{a} \log h(x) d x=-\infty \text {. }
$$

The critical step in the proof of Theorem 4.1 is a choice of test functions $F_{I}$ where the intervals $I$ are tailored to the geometry of $\Omega$. These intervals are provided by

The Good Intervals Lemma. Suppose $g$ is a bounded, strictly positive, monotonically increasing function on an interval $(0, a]$, with

$$
\lim _{x \rightarrow 0+} g(x)=0
$$

and

$$
\int_{0}^{a} \log g(x) d x=-\infty
$$


Then there exists a sequence of intervals $I_{n}-\left(a_{n}, b_{n}\right]$ in $(0, a]$ such that

$$
\begin{gathered}
b_{1}>a_{1} \geq b_{2}>a_{2} \geq \cdots \rightarrow 0, \\
\left|I_{n}\right|=g\left(b_{n}\right),
\end{gathered}
$$

and

$$
\sum_{n=1}^{\infty}\left|I_{n}\right| \log \left|I_{n}\right|=-\infty
$$

In order to preserve the flow of our argument, we postpone the proof of this lemma until $\S 4.11$.

We apply the Good Intervals Lemma to the function $g=\sqrt{h}$, where $h$ is the function described in the first paragraph of this subsection, whose graph forms the boundary of $\Omega$ near the origin. Now $g$ inherits all the hypotheses placed on $h$, including non-integrability of the logarithm, so we may apply the lemma to it, thus obtaining a sequence of consecutive intervals $I_{n}=\left(a_{n}, b_{n}\right.$ ] satisfying conditions (5)-(7) above.

Let $\Gamma_{n}$ be the vertical projection of the interval $I_{n} / 2$ onto $\partial \Omega$. Choose $\delta_{n}$ so that the rectangle $S\left(I_{n}, \delta_{n}\right)$ has height equal to the value of $h$ at the right end-point of $I_{n} / 2$. Since the parametrizing function $h$ for $\partial \Omega$ is monotone increasing on $[0, a]$,

$$
\Gamma_{n}=S\left(I_{n}, \delta_{n}\right) \cap \partial \Omega, \quad \text { where } \delta_{n} \leq 2\left|I_{n}\right|,
$$

as illustrated by Figure 2 (where, for clarity, the subscript " $n$ " has been systematically omitted).

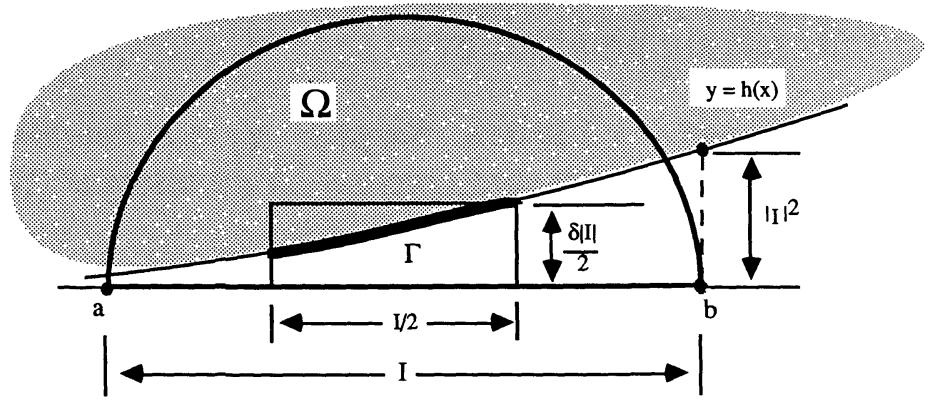

Figure 2. The arc $\Gamma=S(I, \delta) \cap \partial \Omega$ 
Let us write $F_{n}$ for the test function $F_{I_{n}}$. Since $\Gamma_{n} \subset S\left(I_{n}, \delta_{n}\right)$, the pointwise estimate $4.7(\mathrm{~b})$ guarantees that

$$
\left|F_{n}\right|^{2} \geq \frac{1}{\left|I_{n}\right|} e^{-\varepsilon_{n}} \quad \text { on } \Gamma_{n},
$$

where $\varepsilon_{n}=-2 \delta_{n} \log \left|I_{n}\right|$. By $(8)$ and the fact that $\left|I_{n}\right|=\sqrt{h\left(b_{n}\right)} \rightarrow 0$, we have

$$
\varepsilon_{n} \leq 4\left|I_{n}\right| \log \frac{1}{\left|I_{n}\right|} \rightarrow 0 \quad \text { as } n \rightarrow \infty .
$$

Now recall that the univalent mapping $\Phi$ of $\Pi^{+}$onto $\Omega$ extends to a $C^{1}$ homeomorphism-still denoted by " $\Phi$ "-of the closed upper half-plane onto the closure of $\Omega$, with derivative vanishing at no point of the closed half-plane. Let

$$
J_{n}=\Phi^{-1}\left(\Gamma_{n}\right) \quad(n=1,2, \ldots),
$$

so $\left\{J_{n}\right\}$ is a sequence of disjoint real intervals. The boundedness properties of the derivatives of $\Phi$ and $h$ insure that

$$
\left|J_{n}\right| \approx\left|I_{n}\right| \approx \text { length of } \Gamma_{n},
$$

where the notation means, for example, that the sequence of quotients $\left\{\left|J_{n}\right| /\left|I_{n}\right|\right\}$ is bounded, and bounded away from zero, by constants that depend only on $\Phi$, and hence only on the original mapping $\varphi$. Thus we have from (7) and (11) above,

$$
\sum_{n=1}^{\infty}\left|J_{n}\right| \log \left|I_{n}\right|=-\infty
$$

while from (9) and (10),

$$
\left|F_{n} \circ \Phi\right|^{2} \geq \frac{1}{\left|I_{n}\right|} e^{-\varepsilon_{n}} \quad \text { on } J_{n} \text {, where } \varepsilon_{n} \rightarrow 0 \text { as } n \rightarrow \infty \text {. }
$$

4.9. Test functions in action. Having defined the intervals $\left\{I_{n}\right\}$ and $\left\{J_{n}\right\}$, we retreat to the unit disc to finish the argument. In this setting we keep the same notation, letting $I_{n}$ and $J_{n}$ denote the arcs of the circle that correspond, via the Möbius transformation $\tau$ to the original intervals on the line, and writing $f_{n}=F_{n} \circ \tau$. In particular, formulas (11)-(13) of the last section have the following analogues:

$$
\sigma\left(J_{n}\right) \approx \sigma\left(I_{n}\right),
$$

$$
\sum_{n=1}^{\infty} \sigma\left(J_{n}\right) \log \sigma\left(I_{n}\right)=-\infty
$$




$$
\left|f_{n} \circ \varphi\right|^{2} \geq \frac{\text { const. }}{\sigma\left(I_{n}\right)} e^{-\varepsilon_{n}} \quad \text { on } J_{n}, \text { where } \varepsilon_{n} \rightarrow 0 \text { as } n \rightarrow \infty \text {. }
$$

In (3) above, and for the rest of the proof, the symbol "const." will denote a positive constant which may change from one occurrence to the next, but only depends at each occurrence on the original map $\varphi$. For example, in (3) the constant comes from bounds on the derivative of the Möbius transformation $\tau$ in a neighborhood of the point 1 whose size is determined by $\varphi$. Later on, the size of the derivative of $\varphi$ itself near the point 1 will play a role in determining "const."

We also denote by $T\left(I_{n}\right)$ the set in $U$ that corresponds to the semidisc of the same name in the upper half-plane that surmounts the original interval $I_{n}$. The new $T\left(I_{n}\right)$ is the region in $U$ bounded by the arc $I_{n}$ of the unit circle, and the circle through the endpoints of this arc that is perpendicular to $\partial U$.

Now suppose $\psi$ is any holomorphic self-map of $U$, not identically equal to $\varphi$. We emphasize here that while several extra hypotheses have been made on $\varphi$ (e.g., univalence, smoothness at the boundary), no such restrictions are being placed on $\psi$. We will exploit the fact that distinct bounded analytic functions cannot have boundary values too close to each other too often, as quantified by the fact that the logarithm of their difference must be integrable (cf. $\S 1.4)$. Let

$$
E_{n}=\left\{\zeta \in J_{n}:|\varphi(\zeta)-\psi(\zeta)| \geq 4 \pi \sigma\left(I_{n}\right)\right\}
$$

(the lower bound is twice the ordinary arc length of $I_{n}$; had we chosen to stay in the upper half-plane, the length of $I_{n}$ would have sufficed). Then for each $\zeta \in E_{n}$ the image $\varphi(\zeta)$ belongs to $T\left(I_{n}\right)$. Now for $n$ sufficiently large, the diameter of the set $T\left(I_{n}\right)$ is very close to the length of $I_{n}$, and therefore $<4 \pi \sigma\left(I_{n}\right)$, so

$$
\zeta \in E_{n} \Rightarrow \psi(\zeta) \notin T\left(I_{n}\right) .
$$

Thus by 4.7(a) (for the unit disc), if $n$ is sufficiently large, then

$$
\left|f_{n} \circ \psi\right| \leq 1 \text { on } E_{n},
$$

which, along with (3) above (with $n$ sufficiently large), implies that

$$
\left|f_{n} \circ \varphi-f_{n} \circ \psi\right|^{2} \geq \frac{\text { const. }}{\sigma\left(I_{n}\right)} \text { on } E_{n} \text {. }
$$

Upon integrating this last inequality over $E_{n}$ we obtain

$$
\left\|f_{n} \circ \varphi-f_{n} \circ \psi\right\|^{2} \geq \text { const. } \frac{\sigma\left(E_{n}\right)}{\sigma\left(I_{n}\right)} \quad(\text { for } n \text { sufficiently large), }
$$


which yields, because the test functions $\left\{f_{n}\right\}$ are "essentially unit vectors" (inequalities (4) of $\S 4.6$ ),

$$
\begin{aligned}
\left\|C_{\varphi}-C_{\psi}\right\|^{2} & \geq \text { const. } \limsup _{n \rightarrow \infty}\left\|f_{n} \circ \varphi-f_{n} \circ \psi\right\|^{2} \\
& \geq \text { const. } \limsup _{n \rightarrow \infty} \frac{\sigma\left(E_{n}\right)}{\sigma\left(I_{n}\right)} .
\end{aligned}
$$

In view of (1) above, this estimate can also be written

$$
\left\|C_{\varphi}-C_{\psi}\right\|^{2} \geq \text { const. } \limsup _{n \rightarrow \infty} \frac{\sigma\left(E_{n}\right)}{\sigma\left(J_{n}\right)},
$$

so the following lemma will complete the proof that $C_{\varphi}$ is isolated.

4.10. LeMmA. $\lim \sup _{n \rightarrow \infty} \frac{\sigma\left(E_{n}\right)}{\sigma\left(J_{n}\right)}=1$.

Proof. As we mentioned before, the key is that, since $\varphi \not \equiv \psi$, the boundary function $\log |\varphi-\psi|$ is integrable over $\partial U$. Thus, since $|(\varphi-\psi) / 2| \leq 1$ on $\partial U$, we have

$$
\begin{aligned}
-\infty & <\int_{\partial U} \log \left|\frac{\varphi-\psi}{2}\right| d \sigma \\
& \leq \sum_{n} \int_{J_{n} \backslash E_{n}} \log \left|\frac{\varphi-\psi}{2}\right| d \sigma \quad \text { (disjointness of } J_{n} \text { 's) } \\
& \leq \sum_{n} \sigma\left(J_{n} \backslash E_{n}\right) \log \left[4 \pi \sigma\left(I_{n}\right)\right] .
\end{aligned}
$$

Thus

$$
\sum_{n} \sigma\left(J_{n} \backslash E_{n}\right) \log \sigma\left(I_{n}\right)>-\infty,
$$

so in view of $4.9(2)$ we must have

$$
\liminf _{n \rightarrow \infty} \frac{\sigma\left(J_{n} / E_{n}\right)}{\sigma\left(J_{n}\right)}=0
$$

which is equivalent to what we want to show. This completes the proof of the lemma, and with it (lacking only the proof of the "Good Intervals Lemma"), the proof of Theorem 4.1.

4.11. Proof of the "Good Intervals Lemma". In order to avoid having to deal with inequalities where both sides are negative, we work with the function $\log (1 / g(x))$ instead of $\log g(x)$. We define consecutive, disjoint intervals $\left(x_{n+1}, x_{n}\right]$ as follows. First, choose $0<x_{1} \leq a$ 
so that $g\left(x_{1}\right) \leq e^{-2}$. Then proceed by induction: having chosen $x_{1}>x_{2}>\cdots>x_{n}>0$, define a provisional next point by

$$
\xi_{n+1}=x_{n}-g\left(x_{n}\right) \text {. }
$$

If $\xi_{n+1}>0$, and

$$
\int_{\xi_{n+1}}^{x_{n}} \log \frac{1}{g(x)} d x<2 g\left(x_{n}\right) \log \frac{1}{g\left(x_{n}\right)},
$$

then set $x_{n+1}=\xi_{n+1}$, and call the resulting interval $\left[x_{n+1}, x_{n}\right)$ a good interval. Note that the length of a good interval $I$ is just the value of $g$ at its right endpoint, so inequality (1) above can be rewritten:

$$
\int_{I} \log \frac{1}{g(x)} d x<2|I| \log \frac{1}{|I|} \text {. }
$$

If (1) above fails (which includes the possibility that $\xi_{n+1} \leq 0$ ), then there exists a unique point $x_{n+1} \in\left[\xi_{n+1}, x_{n}\right)$ such that

$$
\int_{x_{n+1}}^{x_{n}} \log \frac{1}{g(x)} d x=2 g\left(x_{n}\right) \log \frac{1}{g\left(x_{n}\right)} .
$$

We call the resulting interval $\left[x_{n+1}, x_{n}\right)$ a bad interval.

Clearly the above procedure gives rise to an infinite collection of intervals. Changing the notation a bit, we enumerate the good intervals as $I_{n}=\left(a_{n}, b_{n}\right]$, arranged in order of proximity to the origin:

$$
b_{1}>a_{1} \geq b_{2}>a_{2} \geq \cdots,
$$

and similarly enumerate the bad intervals as $B_{n}=\left(\alpha_{n}, \beta_{n}\right]$.

We claim that the totality of good and bad intervals exhausts $\left(0, x_{1}\right]$. To see this, note that if $I_{n}$ is a good interval, then $g\left(b_{n}\right)=\left|I_{n}\right|$, so pairwise disjointness implies

$$
\sum_{n} g\left(b_{n}\right) \leq \sum_{n}\left|I_{n}\right| \leq b_{1} .
$$

On the other hand, we will show in a moment that for the $n$th bad interval $B_{n}=\left(\alpha_{n}, \beta_{n}\right]$,

$$
g\left(b_{n}\right) \leq e^{-2^{n}} .
$$

The last two inequalities imply that if all the intervals, good and bad, are arranged in "decreasing order", with $\lambda_{n}$ denoting the left endpoint of the $n$th interval, then $g\left(\lambda_{n}\right) \rightarrow 0$ as $n \rightarrow \infty$. Since $g$ is strictly positive on $\left(0, x_{1}\right]$, it follows that $\lambda_{n} \rightarrow 0$; hence, as promised,

$$
\left(0, x_{1}\right]=\text { disjoint union of all the intervals, good and bad. }
$$


To prove estimate (4) we use (3), which asserts that for each bad interval $\left(\alpha_{n}, \beta_{n}\right]$

$$
\begin{aligned}
2 g\left(\beta_{n}\right) \log \frac{1}{g\left(\beta_{n}\right)} & =\int_{\alpha_{n}}^{\beta_{n}} \log \frac{1}{g(x)} d x \\
& \leq\left(\beta_{n}-\alpha_{n}\right) \log \frac{1}{g\left(\alpha_{n}\right)} \quad \text { [monotonicity of } g \text { ] } \\
& \leq g\left(\beta_{n}\right) \log \frac{1}{g\left(\alpha_{n}\right)},
\end{aligned}
$$

where the last line follows from the definition of the left endpoint $\alpha_{n}$ of a bad interval, and the fact that $\log g\left(\alpha_{n}\right)$ is negative. The inequality above yields for each $n$

$$
g\left(\alpha_{n}\right) \leq g\left(\beta_{n}\right)^{2},
$$

which upon iteration shows that

$$
g\left(\beta_{n}\right) \leq g\left(\alpha_{n-1}\right) \leq g\left(\beta_{n-1}\right)^{2} \leq \cdots \leq g\left(\beta_{1}\right)^{2^{n-1}} \leq e^{-2^{n}},
$$

where the final inequality comes from our choice of the point that initiated the selection procedure. This proves (4).

Now we wish to show that there are infinitely many good intervals $\left\{I_{n}\right\}$, and that they satisfy the divergence condition $4.8(7)$. We claim that

$$
\sum_{n} \int_{B_{n}} \log \frac{1}{g(x)} d x<\infty
$$

where we remind the reader that $\left\{B_{n}\right\}$ denotes the collection of bad intervals. Indeed, if $B_{n}=\left(\alpha_{n}, \beta_{n}\right)$, then beginning as in the derivation of (4), we have

$$
\int_{B_{n}} \log \frac{1}{g(x)} d x=2 g\left(\beta_{n}\right) \log \frac{1}{g\left(\beta_{n}\right)} \leq 2^{n+1} e^{-2^{n}},
$$

where the last inequality follows from (4) and the fact that the function $x \log (1 / x)$ is monotone increasing on the interval $\left(0, e^{-1}\right]$ which, by the monotonicity of $g$ and the definition of $b_{1}$, contains all the points $g\left(\beta_{n}\right)$. This, and the disjointness of the intervals in question, proves $(6)$.

Now (5), (6), and the non-integrability of $\log g$ over intervals containing the origin imply that

$$
\sum_{n} \int_{I_{n}} \log \frac{1}{g(x)} d x=\infty
$$


which by $\left(1^{\prime}\right)$ above shows that

$$
\sum_{n}\left|I_{n}\right| \log \frac{1}{\left|I_{n}\right|}=\infty
$$

Thus the desired conclusion is obtained for the collection of good intervals. This completes the proof of the "Good Intervals Lemma," and with it, the proof of Theorem 3.1.

4.12. REMARK. To see that the mappings of Theorem 4.1 are, as advertised, extreme points, suppose first that the image of such a $\varphi$ is an approach region $\Omega(\kappa)$, where $\kappa$ satisfies the hypotheses of Theorem 4.1. Since the $C^{2}$ hypothesis on $\kappa$ leads to a Carathéodory extension of $\varphi$ that is a $C^{1}$ homeomorphism of closures, with nonvanishing derivative, the restriction of $\varphi$ to the unit circle gives a smooth parametrization of the boundary of $\Omega(\kappa)$ under which the logarithmic integral in Theorem 4.1 turns into the one in Theorem 3.1. More generally, if $\varphi$ is any univalent self-map of $U$ whose image locally contains an $\Omega(\kappa)$ of the type discussed above, then the kind of subordination argument used in Lemma 2.1, along with the version of Littlewood's Principle for integral means of subharmonic functions ([13], page 11) shows that $\log (1-|\varphi|)$ is still not integrable. We leave the details to the reader.

5. Closing remarks. We collect here some comments, complements, and open questions suggested by the work of the previous sections.

5.1. Essential isolation. The essential norm of an operator on Hilbert space is its distance to the closed subspace of compact operators:

$$
\|T\|_{e} \stackrel{\text { def }}{=} \inf \{\|T-K\|: K \text { compact on } H\} .
$$

It is not difficult to check that all the isolation results of this paper (Theorem 2.1, Corollary 2.2, and Theorem 4.1) remain true if the operator norm is replaced by the essential norm. The reason for this is that the lower bounds obtained in these results have the form

$$
\|T\| \geq \delta \stackrel{\text { def }}{=} \liminf _{n \rightarrow \infty}\left\|T u_{n}\right\|,
$$

where $T$ is an appropriate difference, or linear combination of composition operators, and $\left\{u_{n}\right\}$ is a sequence of unit vectors that converges weakly to zero. Thus, if $K$ is any compact operator on $H^{2}$, then 
$\left\|K u_{n}\right\| \rightarrow 0$, and we have

$$
\begin{aligned}
\|T-K\| & \geq \liminf _{n \rightarrow \infty}\left\|(T-K) u_{n}\right\|, \\
& \geq \liminf _{n \rightarrow \infty}\left(\left\|T u_{n}\right\|-\left\|K u_{n}\right\|\right), \\
& =\delta,
\end{aligned}
$$

from which it follows that $\|T\|_{e} \geq \delta$.

Thus, all the composition operators we have shown here to be isolated are actually essentially isolated. Is this true of every isolated composition operator?

5.2. Components and compact differences. In the other direction, we pointed out in Remark 3.4(a) that the composition operators we have shown to be not isolated (those induced by maps $\varphi$ that satisfy the non-integrability condition $3.1(*)$ ) actually have compact differences with certain other composition operators which belong to the same component in $\operatorname{Comp}\left(H^{2}\right)$.

This suggests the problem of characterizing the component of a member of $\operatorname{Comp}\left(H^{2}\right)$. The results above suggest a candidate: all composition operators that differ from the given one by a compact operator. Another way of stating our question/conjecture about components is this: is the quotient of $\operatorname{Comp}\left(H^{2}\right)$ by the compact operators totally disconnected?

Further evidence in favor of this answer comes from two sources. First, Proposition 2.2 shows that the compact composition operators all belong to a single component. However we do not know if any non-compact composition operators belong to this component.

Next there is recent work of Barbara MacCluer [19], which shows that whenever two composition operators belong to the same component, then their inducing functions must have the same angular derivative (possibly infinite) at each point of the unit circle. In $\$ 1.10$ we pointed out that if a composition operator is compact, then its inducing map cannot have a finite angular derivative at any point of the unit circle. MacCluer's result extends this conclusion to every composition operator in the component of the compacts.

Recall also from $\S 1.10$ that although non-existence of the angular derivative does not characterize the compact composition operators on $H^{2}$, it does characterize compactness for operators induced by univalent, or even boundedly valent maps. Thus, for example, the component in $\operatorname{Comp}\left(H^{2}\right)$ of the compact composition operators cannot contain any non-compact composition operators induced by such 
maps. Moreover, the work in [19] deals with the essential norm, and shows that if two composition operators either differ by a compact, or lie in the same component, then they must be induced by mappings with identical angular derivatives.

The work of [19] is actually carried out in a general context that includes both $H^{2}$ and the Bergman space $A^{2}$ : the Hilbert space of holomorphic functions square integrable with respect to area measure on $U$. Composition operators also act on $A^{2}$, and for this space the compactness of such operators is equivalent to non-existence of the angular derivative of the inducing map ([18], Theorem 3.5; [25], Corollary 6.11) Thus

\section{$\checkmark \quad$ the compact composition operators on $A^{2}$ form a path component in $\operatorname{Comp}\left(A^{2}\right)$.}

This result provides strong evidence in favor of our conjecture about components, at least in the Bergman space setting. It would be interesting to know if it also holds for $H^{2}$.

5.3. Which composition differences are compact? The discussion above raises the question of characterizing, in some concrete fashion, those pairs of inducing maps for which the corresponding difference of composition operators is compact. For a single composition operator, the answer to the compactness question lies in the asymptotic behavior of the Nevanlinna Counting Function of the inducing map [25]. It would be of interest to obtain a similar result for differences, with perhaps some sort of joint counting function figuring into the problem.

5.4. Which composition operators are isolated? This should perhaps have been the first question of the section. We have pointed out that the obvious conjecture, "extreme points," is necessary, but not sufficient for isolation. As a first step it might be desirable to remove some of the smoothness and monotonicity hypotheses required for our proof of Theorem 4.1, in order to move toward the largest possible class of univalent maps for which the extreme points are exactly the ones that induce isolated composition operators. As we pointed out at the end of $\S 1.8$, the $C^{2}$ hypothesis on contact functions can be weakened somewhat without changing any of our arguments.

5.5. Other values of " $p$." The major results of this paper continue to hold, if minor modifications are made to their proofs, for all the Hardy spaces $H^{p}, 0<p<\infty$. The only real exception is our Hilbert space oriented proof of Berkson's Theorem (Theorem 2.3, Corollary 
2.4). However Berkson's original proof works for all values of $p$ : in fact that is the context in which it takes place.

\section{REFERENCES}

[1] E. Berkson, One-parameter semigroups of isometries into $H^{p}$, Pacific J. Math., 86 (1980), 403-413.

[2] E. Berkson, Composition operators isolated in the uniform operator topology, Proc. Amer. Math. Soc., 81 (1981), 230-232.

[3] E. Berkson and H. Porta, Hermitian operators and one-parameter groups of isometries in Hardy spaces, Trans. Amer. Math. Soc., 185 (1973), 331-354.

[4] P. S. Bourdon and J. H. Shapiro, Cyclic phenomena for composition operators, in preparation.

[5] C. Carathéodory, Theory of Functions of a Complex Variable, Vol II, second English edition, Chelsea, New York, 1960.

[6] J. G. Caughran, Polynomial approximation and spectral properties of composition operators on $H^{2}$, Indiana Univ. Math. J., 21 (1971), 81-84.

[7] J. G. Caughran and H. J. Schwartz, Spectra of compact composition operators, Proc. Amer. Math. Soc., 51 (1970), 127-130.

[8] C. C. Cowen, Composition operators on $\mathrm{H}^{2}$, J. Operator Theory, 9 (1983), 77-106.

[9] _ Subnormality of the Cesàro operator and a semigroup of composition operators, Indiana Univ. Math. J., 33 (1984), 305-318.

[10] _ Linear fractional composition operators on $\mathrm{H}^{2}$, Integral Equations Operator Theory, 11 (1988), 151-160.

[11] C. C. Cowen and T. L. Kriete III, Subnormality and composition operators on $H^{2}$, J. Funct. Anal., 81 (1988), 298-319.

[12] J. A. Deddens, Analytic Toeplitz and composition operators, Canad. J. Math., 24 (1972), 859-865.

[13] P. L. Duren, Theory of $H^{p}$ Spaces, Academic Press, New York, 1970.

[14] J. Garnett, Bounded Analytic Functions, Academic Press, New York, 1981.

[15] P. R. Halmos, A Hilbert Space Problem Book, 2nd ed., Springer-Verlag, New York, 1982.

[16] H. Kamowitz, The spectra of composition operators on $H^{p}$, J. Funct. Anal., 18 (1975), 132-150.

[17] J. E. Littlewood, On inequalities in the theory of functions, Proc. London Math. Soc., 23 (1925), 481-519.

[18] B. D. MacCluer and J. H. Shapiro, Angular derivatives and compact composition operators on Hardy and Bergman spaces, Canad. J. Math., 38 (1986), 878-906.

[19] B. D. MacCluer, Components in the space of composition operators, Integral Equations Operator Theory, 12 (1989), 725-738.

[20] E. Nordgren, Composition operators, Canad. J. Math., 20 (1968), 442-449.

[21] Ch. Pommerenke, Univalent Functions, VandenHoeck \& Ruprecht, Göttingen, 1975.

[22] W. Rudin, Real and Complex Analysis, 2nd ed. McGraw-Hill, New York, 1974.

[23] J. V. Ryff, Subordinate $H^{p}$ functions, Duke Math. J., 33 (1966), 347-354.

[24] H. J. Schwartz, Composition operators on $H^{p}$, Thesis, Univ. of Toledo, 1969.

[25] J. H. Shapiro, The essential norm of a composition operator, Annals of Math., 125 (1987), 375-404. 
[26] J. H. Shapiro and P. D. Taylor, Compact, nuclear, and Hilbert-Schmidt composition operators on $H^{p}$, Indiana Univ. Math. J., 23 (1973), 471-496.

[27] A. Siskakis, Composition semigroups and the Cesàro operator on $H^{p}$, J. London Math. Soc., (2) 36 (1987), 153-164.

[28] _ Personal communication, 1986.

[29] N. Zorboska, Composition operators on weighted Hardy spaces, Thesis, Univ. of Toronto, 1988.

Received November 3, 1988 and in revised form, June 5, 1989. Research supported in part by the National Science Foundation.

Michigan State University

EAst LANSING, MI 48824

AND

UNIVERSITY OF TENNESSEE

KNOXVILLE, TN 37996 


\section{PACIFIC JOURNAL OF MATHEMATICS EDITORS}

\author{
V. S. VARAdarajaN \\ (Managing Editor) \\ University of California \\ Los Angeles, CA 90024-1555-05 \\ Herbert Clemens \\ University of Utah \\ Salt Lake City, UT 84112 \\ Thomas ENRIGHT \\ University of California, San Diego \\ La Jolla, CA 92093
}

R. FINN

Stanford University

Stanford, CA 94305

HermanN FlaschKa

University of Arizona

Tucson, AZ 85721

VAUGHan F. R. Jones

University of California

Berkeley, CA 94720

STEVEN KeRCKHOFF

Stanford University

Stanford, CA 94305
C. C. Moore University of California Berkeley, CA 94720

MARTIN SCHARLEMANN University of California Santa Barbara, CA 93106

\section{HAROLD STARK}

University of California, San Diego La Jolla, CA 92093

\section{ASSOCIATE EDITORS}
R. ARENS
E. F. BECKeNBACH
B. H. NeumanN
F. WolF
(1904-1989)
K. Yoshida (1906-1982)

\section{SUPPORTING INSTITUTIONS}

UNIVERSITY OF ARIZONA

UNIVERSITY OF BRITISH COLUMBIA

CALIFORNIA INSTITUTE OF TECHNOLOGY

UNIVERSITY OF CALIFORNIA

MONTANA STATE UNIVERSITY

UNIVERSITY OF NEVADA, RENO

NEW MEXICO STATE UNIVERSITY

OREGON STATE UNIVERSITY
UNIVERSITY OF OREGON

UNIVERSITY OF SOUTHERN CALIFORNIA

STANFORD UNIVERSITY

UNIVERSITY OF HAWAII

UNIVERSITY OF TOKYO

UNIVERSITY OF UTAH

WASHINGTON STATE UNIVERSITY

UNIVERSITY OF WASHINGTON 


\section{Pacific Journal of Mathematics}

Vol. 145, No. 1 September, 1990

Sheldon Jay Axler and Allen Lowell Shields, Extensions of harmonic and

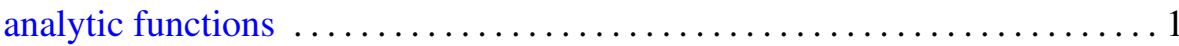

Labib Haddad and Yves Sureau, Les cogroupes et la construction de

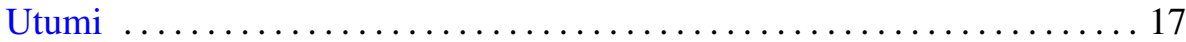

John Hutchinson, Poincaré-Sobolev and related inequalities for submanifolds of $\mathbf{R}^{N}$

Yuk Jaum Leung and Glenn E. Schober, Some coefficient problems and

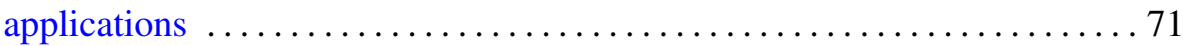

Daniel Ruberman, Seifert surfaces of knots in $S^{4} \ldots \ldots \ldots \ldots \ldots \ldots \ldots \ldots$

Joel Harold Shapiro and Carl Sundberg, Isolation amongst the

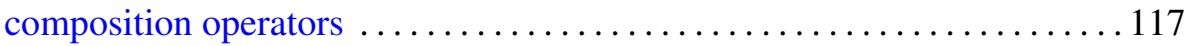

Hans Wenzl, Representations of braid groups and the quantum Yang-Baxter

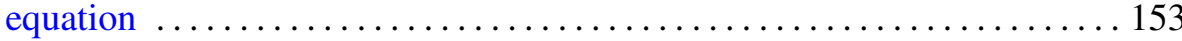

Shuang Zhang, Diagonalizing projections in multiplier algebras and in matrices over a $C^{*}$-algebra 